\title{
Médiévales
}

Langues, Textes, Histoire

68 | printemps 2015

Langues d'Angleterre

\section{Peut-on parler de judéo-anglo-normand ? Textes anglo-normands en écriture hébraïque}

Is there such a Thing as Judeo-Anglo-Norman? Anglo-Norman Texts in Hebrew

Script

David Trotter

\section{OpenEdition}

Journals

Édition électronique

URL : https://journals.openedition.org/medievales/7549

DOI : $10.4000 /$ medievales. 7549

ISSN : $1777-5892$

Éditeur

Presses universitaires de Vincennes

Édition imprimée

Date de publication : 15 juin 2015

Pagination : 25-34

ISBN : 978-2-84292-430-0

ISSN : 0751-2708

\section{Référence électronique}

David Trotter, «Peut-on parler de judéo-anglo-normand ? Textes anglo-normands en écriture

hébraïque », Médiévales [En ligne], 68 I printemps 2015, mis en ligne le 15 juin 2017, consulté le 22 avril 2022. URL : http://journals.openedition.org/medievales/7549; DOI : https://doi.org/10.4000/

medievales.7549 



\section{Peut-on parler de judéo-anglo-normand? Textes anglo-normands en écriture hébraïque}

Les écrits des juifs en Angleterre au Moyen Âge se situent dans deux contextes complémentaires. D'une part, ils constituent des éléments à ajouter à la liste des documents en langue romane rédigés dans les communautés juives, et parfois transcrits en caractères hébreux. Dans le domaine galloroman, le phénomène est également visible dans la France du Nord et en occitan. D'autre part, bien entendu, ces textes font partie du plurilinguisme en Angleterre. Or ils sont assez peu connus des non-spécialistes et n'ont donc pas encore été intégrés dans le débat sur le plurilinguisme anglais, qui apparaît de plus en plus actif depuis à peu près deux décennies. En réalité, nous n'avons pas affaire à des textes proprement dits, mais bien à des mots isolés, c'est-à-dire, à des gloses. Il s'agit donc d'un apparat explicatif qui a été ajouté à d'autres textes et qui a donc un rôle soit pédagogique, soit encore de faciliter la compréhension du glosateur lui-même. D'un certain point de vue, d'ailleurs, l'intérêt de ces matériaux est surtout d'ordre culturel et sociolinguistique, comme témoignages des rapports et des contacts entre les langues. L'apport strictement linguistique est en fait très limité.

Les textes concernés ne sont pas sans poser des problèmes d'interprétation, et même d'attribution. Comme les juifs anglais étaient originaires de Normandie $^{1}$, distinguer entre des textes anglo-normands et normands n'est pas toujours chose facile. De surcroît, il est probable que

1. E. De Visscher, «Hebrew, Latin, French, English: Multilingualism in JewishChristian Encounters», dans J. Jefrerson et A. Putter éd., Multilingualism in Medieval Britain (ca 1066-1520). Sources and Analysis, Turnhout, 2013, p. 89-103; N. GoLB, The Jews in Medieval Normandy. A Social and Intellectual History, Cambridge, 1998, p. 112-114; J. Hillaby, «Jewish Colonisation in the Twelfth Century», dans P. Skinner éd., The Jews in Medieval Britain. Historical, Literary and Archaeological Perspectives, Woodbridge, 2003, p. 16-40 (p. 16). 
les érudits juifs anglais et leurs confrères normands restaient en contact ${ }^{2}$. Très souvent, le seul critère utile est paléographique: devant une écriture anglaise de textes en alphabet latin, l'on sait au moins que le manuscrit aura été copié en Angleterre, ce qui pousse à croire que la langue pourrait bien être insulaire elle aussi ${ }^{3}$.

Or, comme leurs coreligionnaires dans d'autres pays de l'Europe du Nord, les juifs d'Angleterre écrivaient dans la langue du pays: en l'occurrence, l'anglo-normand ${ }^{4}$. Il ne subsiste que vingt-huit livres hébreux de l'Angleterre médiévale ${ }^{5}$ - beaucoup ont été détruits ou exportés au moment de l'expulsion des juifs en 1290 - chiffre qui ne représente qu'un petit échantillon de ce qui a sûrement existé. Vingt-cinq de ceux-ci contiennent des translittérations, des gloses ou des traductions en une autre langue. Il est probable que ces livres aient appartenu à des chrétiens ${ }^{6}$. Il s'agit d'une preuve du contact judéo-chrétien ou, si l'on veut, latin-françaishébreu, qui mérite que l'on s'y attarde.

Le concept de «judéo-anglo-normand» est évidemment dérivé en quelque sorte de celui de «judéo-français». Depuis 1973, les romanistes ont renoncé à croire au judéo-français en tant que langue spécifique qui aurait été parlée par les juifs français au Moyen Âge et qui aurait été un dialecte distinct de l'ancien français. À la différence du judéo-espagnol parlé par les Sépharades, le français des juifs du Moyen Âge, s'il possédait des éléments de vocabulaire différents, n'était pas une langue ou un dialecte indépendant. Ainsi, si j'utilise dans cette contribution le terme «judéo-anglo-normand», ce n'est nullement avec le sens de «variété particulière de l'anglo-normand utilisée par les juifs». Si le judéo-français est une «langue fantôme»

2. R. StaceY, «The English Jews under Henry III», dans P. Skinner éd., The Jews in Medieval Britain..., p. 41-54 (p. 47-48).

3. Je remercie vivement Marc Kiwitt de ses observations très pertinentes (communication privée) sur cette question dans le cas du ms. Valmadonna 1, et du texte de Moïse ibnEzra. M. Kiwitt souligne que, d'une part, le consonantisme hébreu n'est pas univoque pour représenter le français et que, d'autre part, ces textes font partie d'une tradition qui a déjà sa propre histoire et qui a donc un lexique qui lui est aussi propre. Tout cela réduit considérablement la certitude avec laquelle on peut tenter de localiser de tels textes. Voir aussi: M. KıwıTT, «Les glossaires bibliques hébraïco-français du XIII ${ }^{\mathrm{e}}$ siècle et le transfert du savoir profane», dans S. DörR et R. WiLhelm éd., Transfert des savoirs au Moyen Âge. Wissenstransfer im Mittelalter, Heidelberg, 2008, p. 65-80; G. Bos, G. Mensching et J. Zwink, «A late medieval Hebrew-French glossary of Biblical animal names», Romance Philology, 63 (2009), p. 71-94.

4. E. De Visscher, «Hebrew, Latin, French, English...», p. 93; K. Fudeman, Vernacular Voices. Language and Identity in Medieval French Jewish Communities, Philadelphie/ Oxford, 2010, p. 89-123.

5. P. SkInNER, The Jews in Medieval Britain..., p. 6.

6. E. De Visscher, «Hebrew, Latin, French, English... », p. 94. 
selon l'expression de Menachem Banitt, le «judéo-anglo-normand » l'est également, sinon plus ${ }^{7}$.

La définition du terme «judéo-anglo-normand» n'est pas pour autant entièrement réglée. En tête du glossaire des mots français du Dictionnaire de la Bible hébraïque de l'abbaye de Ramsey, Geneviève Hasenohr écrit (en expliquant les abréviations utilisées): «Jfr (judéo-français), enfin, signale que le mot (la forme et le/les sens) a déjà été relevé dans des textes écrits par des juifs en France du Nord entre le XI ${ }^{\mathrm{e}}$ et le $\mathrm{XIV}^{\mathrm{e}}$ siècle $^{8}$. » Des mots, donc, qui semblent appartenir à un lexique spécialisé qui ne se trouve que dans ces écrits. Il s'agit à mon avis d'une définition en réalité assez trompeuse. Souvent ce sont des mots qui sont utilisés par les juifs, et ne sont utilisés que par eux, parce qu'il s'agit d'un lexique insolite concernant les écrits religieux hébreux. Il est évident aussi qu'un pourcentage important - presque la totalité - de ce qui a survécu des écrits juifs de l'Angleterre médiévale porte sur la littérature religieuse, ce qui implique un vocabulaire assez spécialisé 9 .

La définition adoptée par les spécialistes qui travaillent sur l'ancien français rédigé au sein des communautés juives est plus limitée et plus précise : le judéo-français est le français écrit en caractères hébreux. En le transposant dans le monde anglo-normand, l'on s'aperçoit que le nombre de textes concernés est lui aussi assez limité. Or les écrits en anglonormand provenant des juifs anglais peuvent être divisés en trois catégories principales, dont les deux premières ne correspondent pas à la définition proposée ici et ne sont rappelés ici que pour compléter le tableau:

1. Textes juridiques ou «starrs », rédigés en anglo-normand (alphabet latin) mais qui ne sont pas différents d'autres textes anglo-normands du même genre, si ce n'est pour un nombre très réduit de lexèmes dont estare lui-même ${ }^{10}$.

7. M.BAnITT, «Une langue fantôme : le judéo-français », Revue de Linguistique Romane, 27 (1963), p. 17-294; cf. (pour un traitement plus général) M. SALA, «Die romanischen Judensprachen. Les langues judéo-romanes», dans G. Holtus, M. Metzeltin et C. Schmitt éd., Lexikon der Romanistischen Linguistik, 7, p. 372-395 [en français].

8. J. Olszowy-Schlanger et al., Dictionnaire hébreu-latin-français de la Bible hébraïque de l'abbaye de Ramsey (XIII's.), Turnhout, 2008, p. 245.

9. Sur le judéo-français, lire à présent le chapitre 1.8 de M. KiwıtT, Les Gloses françaises du glossaire biblique B. N. hébr. 301. Édition critique partielle et étude linguistique, Heidelberg, 2013.

10. J. RigG, Select Pleas, Starrs, and Other Records from the Rolls of the Exchequer of the Jews, A.D. 1220-1284, Londres, 2001 (Selden Society, 15). 
2. Gloses en alphabet latin à des ouvrages religieux en hébreu, par exemple (1) le Dictionnaire de l'Abbaye de Ramsey ${ }^{11}$, ou (2) le psautier annoté du manuscrit Paris, BnF, hébreu $113^{12}$.

3. Textes hébreux avec gloses ou commentaires en anglo-normand, en caractères hébreux: (3) le manuscrit Valmadonna $1^{13}$; (4) le lapidaire de Berakhyah Ben Natronai ha-Nakdan ${ }^{14}$; ou (5) le manuscrit un texte de Moïse ibn Ezra d'Oxford (Oxford, Bodley Or. 135) ${ }^{15}$, qui a la particularité de présenter des gloses anglo-normandes en alphabet hébreu, lesquelles sont par la suite traduites en latin (alphabet latin).

\section{Le Dictionnaire de l'Abbaye de Ramsey}

Ce dictionnaire du troisième quart $\mathrm{du} \mathrm{XIII}^{\mathrm{e}}$ siècle est la création des moines bénédictins de l'abbaye de Ramsey. Voici quelques exemples pris au hasard des mille gloses anglo-normandes qu'il contient:

sub PE, f $44 v^{\circ} a$ :

105. פַח Peten

Limen, gallice «suil» $\left(A N D^{16}\right.$ sub soeil $)$

106. פְּרִ Paresez

Expansio, gallice «estendement» (AND Ø)

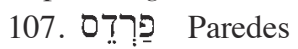

Paradisus, ebreus gallice «gardin» (AND sub gardin, sens à ajouter)

sub ALEPH, $\mathrm{f}^{\circ} 89 \mathrm{v}^{\circ}$

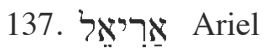

Dei leo uel Mansiuncula, gallice «maneret» (AND $\varnothing, c f$. maner? «ce diminutif est un hapax», GlossRamsey O 255c)

140.

Capsella, gallice «escrin » (AND sub escrin)

11. J. Olszowy-Schlanger et al., Dictionnaire hébreu-latin-français...

12. J. Olszowy-Schlanger, Les Manuscrits hébreux dans l'Angleterre médiévale : étude historique et paléographique, Paris/Louvain, 2003, p. 19-22.

13. M. BeIT-Arié, The Only Dated Medieval Hebrew Manuscript Written in England (1189 CE) and the Problem of Pre-expulsion Anglo-Hebrew Manuscripts, Londres, 1985 [appendice par M. Banitt sur les 14 gloses anglo-normandes, p. 29-31].

14. Berakhyah ben Natronai ha-Nakdan, Sefer Ko'ah ha-Avanim (On the Virtue of the Stones). Hebrew Text and English Translation. With a Lexicological Analysis of the Romance Terminology and Source Study, éd. G. Bos et J. Zwink, Leyde/Boston, 2010.

15. E. BoEHMER, «Un vocabulaire hébraïco-français», Romanische Studien, 1 (18711875), p. 163-220; cf. J. Olszowy-Schlanger, Les Manuscrits hébreux ..., p. 33.

16. Les références abrégées en $A N D$ renvoient à l'Anglo-Norman Dictionary en ligne, consultable à l'adresse suivante: http://www.anglo-norman.net. 


\section{3. אִרוּ Aureueh}

Stabulum uel Presepe, gallice «mangeure» (AND mangure)

La structure des articles est donc: mot hébreu de la Bible, en hébreu; translittération en alphabet latin; explication en latin (ou glose); et, pour un millier de mots au total, glose en anglo-normand (il y a aussi trois gloses moyenanglaises). Visiblement donc, un texte qui ajoutera des éléments à l'AND, sans que ceux-ci soient nécessairement des lexèmes particulièrement liés à l'hébreu ou à la littérature religieuse juive. Mais «maneret » par exemple est important: c'est un hapax précieux.

\section{Le Psautier annoté du manuscrit Paris, BnF, hébreu 113}

Ce manuscrit est indubitablement au moins en partie d'origine anglaise, non seulement à cause de la main anglaise qui aurait ajouté des gloses latines en alphabet latin (second quart du XIII ${ }^{\mathrm{e}}$ siècle), mais aussi parce qu'il comporte $\left(\mathrm{f}^{\circ} 137 \mathrm{v}^{\circ}\right)$ un alphabet runique ${ }^{17}$. Ce sont bien entendu des éléments qui pourraient avoir été ajoutés en Angleterre sur un manuscrit copié en France, les runes étant en tout cas d'une main différente de celle qui a copié les autres textes. Comme exemple de la procédure suivie dans le texte du psautier, voici un détail du folio $4 v^{\circ}$, où l'on lit, d'une part, au-dessus de la ligne 3, la glose latine «una hora», et, d'autre part, audessous de la même ligne mais correspondant bien entendu à un mot hébreu

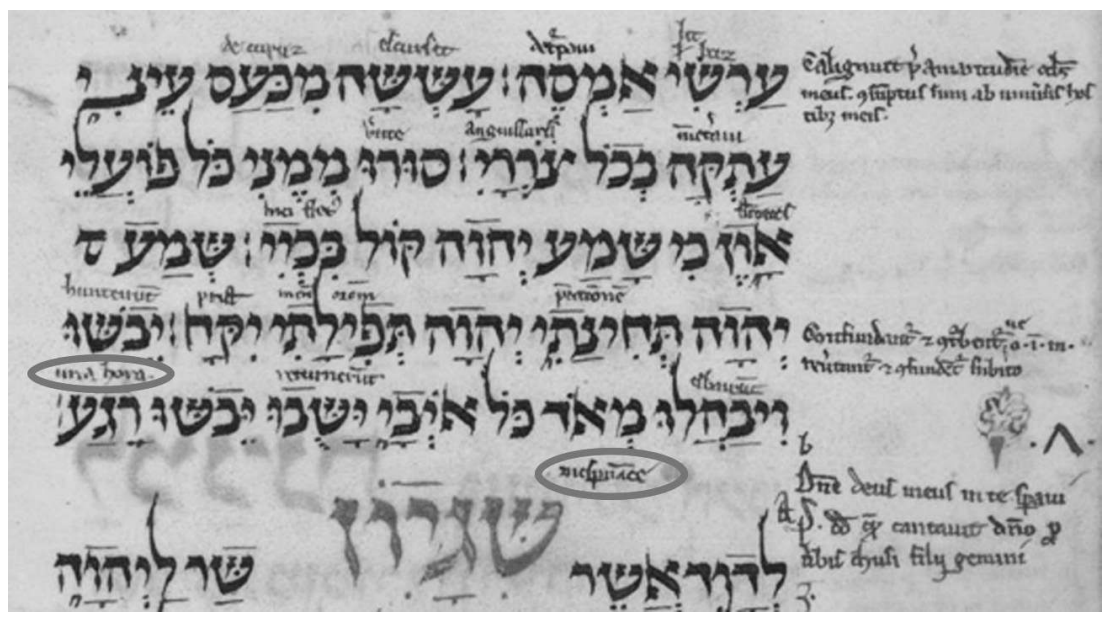

Fig. 1. Le Psautier annoté du manuscrit Paris, BnF, hébreu 113.

17. J. Olszowy-Schlanger, Les Manuscrits hébreux..., p. 181; G. Dahan, «Deux Psautiers hébraïques glosés en latin», Revue des études juives, 158 (1999), p. 61-87. 
différent, la glose française «mespernance». Les deux mots sont encerclés sur l'image ${ }^{18}$.

C'est un texte intéressant surtout pour sa confection, qui n'ajoute peut-être pas grand-chose au niveau linguistique, mais qui mériterait une étude de la part d'un spécialiste compétent.

\section{Le manuscrit Valmadonna 1}

Le manuscrit Valmadonna, antérieur à 1189, est probablement d'origine anglaise. Surtout, la partie qui nous intéresse (des gloses sur les noms d'oiseaux impurs de Lévitique ${ }^{19}$ ) semble d'origine anglo-normande, puisqu'elle contient au moins un mot français qui n'est pas connu en dehors du domaine anglo-normand. Même si une origine normande n'est pas tout à fait exclue ${ }^{20}$, l'élément le plus probant semble être le mot malve (mauve), inconnu sauf en anglo-normand, et pour cause - il s'agit d'un mot d'origine anglo-saxonne $(\text { maew })^{21}$. La seule attestation «continentale» de mauve se trouve dans le Roman des Deduis de Gace de la Buigne, «commencé en 1359 en Angleterre» selon le Dictionnaire étymologique de l'Ancien Français (GaceBuigneB). Pour Norman Golb, «il n'y a aucune raison de penser que [Marie de France] ait eu connaissance du mot malve ailleurs qu'en Normandie [...] il serait difficile de penser que [Philippe de Thaon] ait eu connaissance du mot malve pour la première fois après son arrivée en Angleterre», argument loin d'être convaincant ${ }^{22}$. Si le mot n'est autrement attesté qu'en Angleterre, il semble logique de croire que c'est là où le copiste l'aura trouvé. La transcription proposée par Menachem Banitt ne me semble pourtant pas entièrement correcte : sauf erreur de ma part, il faut lire dans[שאלב[א] [שריצ calve souriz (assez anglo-normand) et non pas chalve.

18. Manuscrit consulté sur le site Gallica: http://gallica.bnf.fr/ark:/12148/btv1b60004143, le 29 septembre 2013.

19. Sur lesquels on verra aussi D. TRotTER, «Science avec conscience: réflexions sur le lexique scientifique et le DMF [Dictionnaire de Moyen Français]», dans F. Duval éd., La «logique» du sens: de la sémantique à la lexicographie. Autour des propositions de Robert Martin, Metz, 2011, p. 281-299 (p. 288) et ID., «Tote manere d'oiseaus: les noms d'oiseaux en anglo-normand», Cahiers de lexicologie, 103 (2013), p. 125-143. Le mot malve n'est pas sans poser de problèmes car l'étymon maew a fourni deux mots en principe distincts mauve et mauviz, mais qui, en anglo-normand, sont souvent confondus: voir AND sub mauve et mauviz.

20. N. Golb, The Jews in Medieval Normandy..., p. 457; J. Olszowy-Schlanger, Les Manuscrits hébreux..., p. 238.

21. Französisches etymologisches Wörterbuch 16,495b; Dictionnaire de Godefroy $5,207 \mathrm{c}$; Trésor de la Langue française 5,108.

22. "There is no reason to believe that [Marie de France] learned the term malve anywhere else than in Normandy [...] one would be hard-pressed to believe that [Philippe de Thaon] first learned the term malve after he had come to England» (N. GoLB, The Jews in Medieval Normandy..., p. 462). 
Comme dans le cas de מאלב]אז, malve, la question de la vocalisation ou non de [ł] reste ouverte. Voici la liste des gloses avec transcription selon le système romaniste :

\begin{tabular}{|c|c|c|c|c|}
\hline & $\begin{array}{l}\text { Transcription } \\
\text { Banitt }\end{array}$ & Oiseau & $\begin{array}{l}\text { Translittération } \\
\text { «romaniste»* }\end{array}$ & $\begin{array}{l}\text { Graphie en } \\
\text { hébreu }\end{array}$ \\
\hline 1 & ègle & aigle & 'eYGLə' & 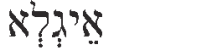 \\
\hline 2 & escofle & écoufle & ’əŠQW[PL'] & אִשִׁקופטלא] \\
\hline 3 & orfraie & balbuzard pêcheur & 'WRPR[YY'] & אורפר] [ייא] \\
\hline 4 & rojas & faucon crécerelle & RWYYŠ & רויישי \\
\hline 5 & jai & geai & YaYa'iY & יהיאי \\
\hline 6 & espervèr & épervier & 'ŠPR[WWYR] & אשפר]וויר] \\
\hline 7 & ostoir & autour & 'WŠṬ[WYR] & אושט][יר] \\
\hline 8 & voltor & vautour & WWLṬW[R] & וולטטור \\
\hline 9 & rosiniol & rossignol & RWŠYN[YWL] & רושינ[יין][ \\
\hline 10 & çuète & chouette & ȘW'YṬ & צואיט \\
\hline 11 & estornèl & étourneau & 'ŠṬ[WRNYL] & אשטי[רביל] \\
\hline 12 & herupe & huppe & HəRWPə’ & חְרוּרְּא \\
\hline 13 & chalve soriç & chauve-souris & Q'LB['] [ŠWRYȘ] & \\
\hline 14 & malve & mouette & M'LB['] & מאלבבאיאי \\
\hline
\end{tabular}

*Voir G. Bos et J. Zwink, Berakhyah Ben Natronai ha-Nakdan..., p. 87-88. Les consonnes de l'hébreu sont rendues par des majuscules, les signes de vocalisation (là où ils apparaissent) par des minuscules.

Ici encore, c'est un texte qui est peut-être intéressant par sa date (avant 1189), mais dont la faible quantité de mots anglo-normands réduit l'importance. En même temps, l'emploi des caractères hébreux pour la transmission des gloses anglo-normandes n'est pas sans intérêt sur le plan culturel et sociolinguistique. Cette pratique semble due à un copiste qui transcrivait en hébreu ce qu'il entendait ou lisait en anglo-normand ; un juif, donc, francophone.

\section{Le Lapidaire de Berakhyah Ben Natronai ha-Nakdan}

Ce texte, un lapidaire composé vers 1300 par Berakhyah ben Natronai ha-Nakdan, juif résidant en France mais qui a probablement passé du temps en Angleterre, est conservé dans un manuscrit d'Oxford (Bodleian Library, Can. Or. 70), où il a survécu dans une copie du XIV siècle, dans la marge d'un texte liturgique. Selon les éditeurs, le copiste «ne connaissait que très peu l'hébreu et n'a guère compris le texte ${ }^{23}{ }^{2}$, ce qui entraîne un

23. Berakhyahben Natronaiha-Nakdan, Sefer Ko'aḥ ha-Avanim..., p. 6. 
grand nombre d'erreurs. De nouveau, il y a incertitude quant à l'origine du manuscrit (insulaire ou continentale?), et selon les éditeurs, «presque tous les termes romans qui ont été identifiés comme de l'ancien français peuvent aussi être interprétés comme de l'anglo-normand ${ }^{24}$ », à quelques exceptions près :

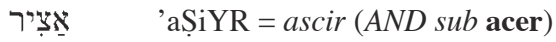

$$
\begin{aligned}
& \text { וינש } \quad W Y N S \check{~=~ v e n e s ~(A N D ~ s u b ~ v e i n e) ~}
\end{aligned}
$$

où les graphies indiqueraient plutôt l'anglo-normand ${ }^{25}$. C'est un critère à mon sens assez peu sûr étant donnée l'instabilité des graphies de l'ancien français et surtout de l'anglo-normand.

Il existe aussi dans ce texte des mots qui ne sont documentés qu'en anglo-normand ${ }^{26}$ :

DeYṬ’ (Bos / Zwink 2010, 103). Inexact: date est également attesté en français, mais tardivement (1476), $c f$. Gdf 2,423c; Du Cange 8,383c; TL 2,1198; AND sub date

פִידוֹרַטַש PiYDWoRaṬaŠ = pedoretes (Bos/Zwink 2010, 104); AND sub pedoretés (une seule citation)

שוֹלְנִיטֶי Solente (Bos/Zwink 2010, 109); OED subs ilenite

אוֹקציביאן ('WoQȘiYa'N) «is, because of the vowelization [sic], rather Angl.Nor. occian, 'ocean, sea', than O. Fr. oc(c)ean» (Bos/Zwink 2010, 92); AND sub occean*

קורלירַא QoWLeYRa' «has to be read as Angl.-Nor. colire» (Bos/Zwink 2010, 105); AND sub collirie

* L'article «ocean » de l'Anglo-Norman Dictionary est encore à développer.

Parmi ces mots, évidemment, trois (pedoretes, solenite, colire) relèvent du domaine des lapidaires, domaine où l'anglo-normand (grâce à la tradition des textes remontant en dernière analyse à Marbode) est surreprésenté et qui sont en tout cas des latinismes assez flagrants. Ce sont en quelque sorte de «faux anglo-normandismes» qui auraient pu également exister sur le continent et qui n'ont à vrai dire rien de définitivement insulaire. 


\section{Le glossaire des bouts rimés de Moïse ibn Ezra d'Oxford}

Ce texte, également conservé dans un manuscrit de la Bodléienne (Oxford, Bodley Or. 135) ${ }^{27}$, comporte des homonymes (en fin de verset) de l'ouvrage de Moïse ibn Ezra, Sefer ha-Anak.

Les gloses françaises (elles-mêmes traduites en latin) traduisent les homonymes hébreux du Tarschisch de Moïse ibn Ezra, poème du début du $\mathrm{XII}^{\mathrm{e}}$ siècle et dont les couplets riment par des homonymes; le manuscrit d'Oxford est de la première moitié du XIII ${ }^{\mathrm{e}}$ siècle. Le texte hébreu (l'homonyme dont il est question) est bien entendu à droite sur la page; il est traduit en anglo-normand (en alphabet hébreu) au centre de la page: cette glose est ensuite traduite en latin (alphabet latin). Voici un exemple du processus : ד du texte hébreu est rendu par la glose anglo-normande (en caractères hébreux) מאמיילה, traduit en latin comme mamilla (Eduard Boehmer transcrit page 170 par erreur mamila).

Voici la partie du manuscrit concernée ${ }^{28}$ :

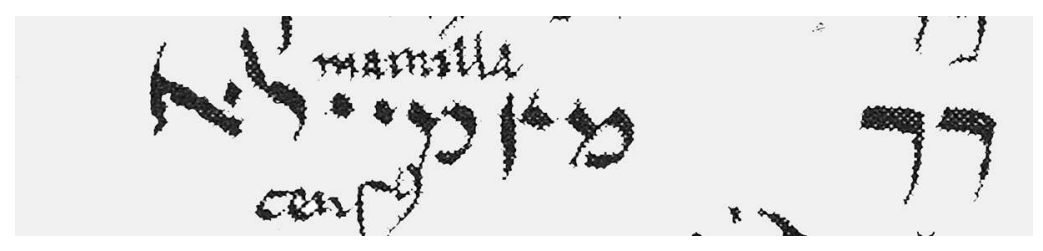

Fig. 2. Exemple d'une glose dans le texte de Moïse ibn Ezra

Le texte tel qu'il apparaît dans le manuscrit peut être présenté de la manière suivante:

$\begin{array}{lllll}\text { glose en caractères hébreux } & \text { Boehmer } & \text { «romanistes» } & \text { Latin } & \text { hébreu } \\ \text { מאמיילה } & \text { mamiele } & \text { M'MYYL' } & \text { Mamilla } & \text { דT }\end{array}$

Du point de vue strictement lexical, ou lexicographique, les textes conservés des juifs d'Angleterre au Moyen Âge ne fournissent que relativement peu de renseignements nouveaux. Leur importance est surtout d'ordre sociolinguistique et culturel. Ils témoignent de rapports entre juifs et chrétiens et de contacts linguistiques, peut-être limités à des groupes très réduits et à des «spécialistes», mais néanmoins importants. Comme les juifs de la France du Nord, les juifs anglais rédigeaient au moins des bribes de textes en anglo-normand, mais en caractères hébreux. Pour ce faire, donc, ils comprenaient l'anglo-normand et ils avaient une idée des

27. E. BoeHMER, «Un vocabulaire hébraïco-français...».

28. Feuillet reproduit dans J. Olszowy-Schlanger, Les Manuscrits hébreux..., p. 260. 
correspondances entre le phonétisme de l'anglo-normand, et l'alphabet hébreu. C'est un élément à ajouter au phénomène du plurilinguisme en Angleterre au Moyen Âge.

David Trotter - Aberystwyth University, Department of European Languages

\section{Peut-on parler de judéo-anglo-normand? Textes anglo-normands en écriture hébraïque}

L'article passe en revue les témoignages (limités) d'écrits liés à la communauté juive d'Angleterre entre la Conquête normande et l'expulsion de 1290. Les textes sont de trois types: 1 . des starrs, documents juridiques rédigés en anglo-normand et qui ne présentent que très peu de différences avec les textes comparables de caractère administratif et juridique rédigés en anglonormand en dehors de la communauté juive ; 2 . des gloses, en alphabet latin, sur des textes hébreux et 3 . des textes anglo-normands (qui sont aussi des gloses) en caractères hébraïques : seuls ces derniers correspondent à ce que l'on entend aujourd'hui par " judéo-français ». La documentation anglonormande est très limitée mais elle fournit néanmoins des renseignements sur les contacts entre juifs et chrétiens. Les documents anglo-normands sont ainsi à la fois une pièce à ajouter au puzzle des textes romans en écriture hébraïque, et un élément du paysage plurilingue de l'Angleterre médiévale.

Angleterre - Bible hébraïque - écriture hébraïque - gloses - hébreu - juifs

\section{Is there such a Thing as Judeo-Anglo-Norman? Anglo-Norman Texts in Hebrew Script}

The article reviews the limited evidence for writings associated with the Jewish community in England between the Conquest and the expulsion of the Jews in 1290. The texts are of three types: 1 . starrs or legal documents, written in Anglo-Norman and displaying very limited divergence from other comparable administrative/legal documents in Anglo-Norman; 2 Romanalphabet glosses to Hebrew texts and 3. Anglo-Norman texts (in fact, glosses) in Hebrew characters. Only the last conform to the definition now generally accepted of "Judeo-French ». The Anglo-Norman textual evidence is very limited but it nevertheless provides information about JewishChristian contact. The Anglo-Norman documents are thus at once a piece in the jigsaw of Romance texts in the Hebrew alphabet, and an element in the multilingual landscape of medieval England.

England - Glosses - Hebrew - Hebrew Bible - Hebrew script - Jews 


\section{Langues d'Angleterre,}

coordonné par Alban Gautier et Jean-Pascal Pouzet

5 Alban Gautier et Jean-Pascal Pouzet

Les langues de

l'Angleterre médiévale : au-delà du bilinguisme

\section{David Trotter}

Peut-on parler de judéoanglo-normand?

Textes anglo-normands en écriture hébraíque

35 Christopher Lucken

Le beau français

d'Angleterre. Altérité de l'anglo-normand et invention du bon usage

57 Aude Mairey John Gower ou le multilinguisme en action

73 Catherine Nall et Daniel Wakelin Le déclin du multilinguisme dans The Boke of Noblesse et son Codicille de William Worcester
Essais et recherches

93 Donatella Nebbiai

Les livres de Jean

Durand († 1416),

"physicien »

et astrologue

\section{Lucie Laumonier}

En prévision des vieux

jours: les personnes âgées à Montpellier à la fin du Moyen Âge

\section{Points de vue}

147 François Foronda Procès politiques: une manie française?

161 Clément Lenoble Monnaie, valeur et citoyenneté chez Olivi et Eiximenis. "Moralisation de l'économie » ou "économie politique" médiévale?

181 Notes de lecture 205 Livres reçus 